\title{
Association of preretinal hemorrhage with the presence and severity of retinopathy of prematurity in high-risk patients
}

\author{
Daniel Garza-Cantú, Saria Sánchez-Álvarez, David Ramón Palacios-Barragán and \\ Gerardo del Carmen Palacios-Saucedo \\ Ophthalmology Department and Research Division, Unidad Médica de Alta Especialidad No. 25, Instituto Mexicano del Seguro Social, Monterrey, \\ N.L., Mexico
}

\begin{abstract}
Objective: To assess whether preretinal hemorrhage $(P R H)$ is associated with the presence and severity of retinopathy of prematurity $(R O P)$ in high-risk patients. Methods: Prospective cohort study, patients referred to the Department of Ophthalmology for ROP screening during October-November 2016 were evaluated weekly on 4 occasions to assess the relationship with PRH and the development of ROP as well as degree of severity associated. We used absolute, median frequencies with minimum and maximum values, $\chi^{2}$ test and Mann-Whitney $U$-test, as well as relative risk with $95 \%$ confidence interval. Results: $A$ total of 30 patients, in the first week 11 females (36\%) and 4 males (13\%) had PRH; in the $2^{\text {nd }}$ week 13 patients (43\%) presented PRH and 14 (46\%) developed ROP; on the $3^{\text {rd }}$ week there were no changes; in the last week 8 presented HPR (26\%) and 11 patients (36\%) with ROP. Stage I severity occurred more frequently. Statistical significance $(p=0.040)$ was found in the presence of PRH and the development of ROP in the first week with. Conclusions: HPR is a risk factor for the development of ROP during the first weeks of life and is associated with stages of mild severity.
\end{abstract}

KEY WORDS: Preretinal hemorrhage. Retinopathy of prematurity. Severity.

\section{Introduction}

Retinopathy of prematurity (ROP) is defined as a progressive retinal vasoproliferative disorder that occurs exclusively in premature infants ${ }^{1}$. Worldwide, incidences ranging from $12 \%$ to $78 \%$, at any of its stages, are reported in children born with less than 1500-g weight ${ }^{2-4}$.

Associated systemic factors include vascular immaturity, state of hypoxia, gestational age, multiple pregnancy, respiratory distress, apnea, acidosis, bradycardia, anemia, sepsis and transfusions ${ }^{5,6}$.

ROP has an international classification that considers three concentric zones that describe the retina from its posterior to the anterior portion (zone), circumferential extension (time zone or meridians) and seriousness (stage) with presence or absence of "plus" disease:

- Zone I: with the optic disc as center, and papilla-fovea double distance, the formed circle is zone I.

- Zone II: starts at zone I border and extends nasally towards the ora serrata with the corresponding temporal area or to the equator.

- Zone III: starts at zone II border and is the remaining retinal area towards the temporal sector. It is the last zone to complete vascularization for retinal circulation full development.

ROP extension is documented according to the number of time zones involved. There are five stages: 
- Stage I or demarcation line: a demarcation line is observed between the retinal avascular and vascular areas.

- Stage II or crest: the demarcation line grows until occupying a volume, and has height and width to form a crest above the retinal plane. Small blood vessels flakes or plumes can be also observed, usually called "popcorn", located posterior to the crest.

- Stage III or fibrovascular proliferation: extraretinal fibrovascular extending from the crest to the vitreous body is observed; it can be continuous or not, and it is localized posterior to the crest.

- Stage IV or subtotal or partial retinal detachment: partial, exudative or traction retinal detachment is observed. It is divided in A when it does not involve the macula, and $B$ when there is.

- Stage V or complete retinal detachment: complete retinal detachment is observed, with leukocoria being clinically evident.

In addition, there are seriousness variants, such as "plus disease", which is defined as venous dilation and arterial tortuosity of the posterior pole vasculature. The "pre-plus" disease is also a vascular alteration that manifests itself as higher-than-normal dilation and tortuosity, but less intensely than in disease plus.

Anterior progressive ROP (formerly known as "rush" disease), a rare, rapidly progressive presentation form that is observed at the posterior pole as serious plus disease and plain intraretinal neovascularization, which can easily progress to stage IV and produce total blindness if not early treated ${ }^{7}$. The exploration technique has to be carried out under pharmacological pupillary dilation and indirect ophthalmoscopy with 28-diopter lens. Ophthalmic dilation solutions based on cyclopentolate $(0.5 \%)$ and phenylephrine $(2.5 \%)$ are generally used, and are applied every 15 minutes for three times ${ }^{2,7}$. The American Academy of Pediatrics establishes that infants with a birth weight of $1500 \mathrm{~g}$ or gestational age shorter than 30 weeks of gestation, as well as certain particular cases with birth weight of 1500-2000 g or gestational age longer than 30 weeks of gestation with unstable clinical status, should be assessed for ROP. Screening should be carried out 4 weeks after birth? ${ }^{7}$. Once ROP has been staged, the different existing treatment should be evaluated. Cryotherapy consists in the use of a probe to freeze avascular retina areas and prevent or decrease the risk of retinopathy-secondary retinal folding or detachments: however, owing to the high risk of complications, such as inflammation, and to the need of general anesthesia in pediatric patients, it is not considered the first choice of treatment anymore ${ }^{8}$. Indirect laser photocoagulation has been shown to be the first line treatment (gold standard). It consists of an indirect infrared system that produces hypoxic retina ablation turning it into anoxic retina, with a subsequent reduction of the vasogenic stimulus, the formation of abnormal vessels and disease progression ${ }^{9}$. Drugs that block the endothelial growth factor or anti-vascular endothelial growth factor (VEGF) are treatments that are applied by intravitreous injection ${ }^{10}$. Surgical treatment is reserved for cases of highly advanced disease, such as stages 4 and 5 . Best results have been shown to be obtained at stage $4 a$, and the procedures that can be offered include vitrectomy and cerclage. In stage 5 cases, vitrectomy and lensectomy or open vitrectomy can be considered; however, from stage $4 \mathrm{~B}$ on there is high risk of total visual loss after surgical management, and therefore the choice of surgery or patient follow-up and observation becomes debatable ${ }^{11}$.

ROP is a pathology that is is generally self-limited, with an average duration of 15 weeks encompassed from the onset of the disease until the period of its inactivation ${ }^{12}$. Finally, the risk factors that are closely related to the development of complications, which in addition are part of the purpose of this study, include the presence of vitreous hemorrhage, and in particular preretinal hemorrhage $(\mathrm{PRH})$. Retinal hemorrhage is not an uncommon finding in ROP; it has been reported at an incidence ranging from $5 \%$ to $33 \%$ in patients with any ROP stage $^{13}$. The purpose of this study consisted in assessing whether $\mathrm{PRH}$ is associated with the presence and severity of ROP in high risk patients.

\section{Method}

The study was carried out in premature infant patients seen at the Ophthalmology Department of the No.25 High-Specialty Medical Unit (UMAE - Unidad Médica de Alta Especialidad) of the Mexican Institute of Social Security, in Monterrey, Nuevo León, for ROP screening during October and November 2016. Premature patients with less than 30 weeks of gestation at birth and with more than 30 days of having been born at assessment, with low weight for gestational age below $1500 \mathrm{~g}$, and with risk factors for ROP, such as use of oxygen therapy, were included. The study underwent evaluation by the medical unit ethics committee and its authorization was obtained, in addition to obtaining informed consent for pediatric patient authorized and signed by the legal guardian of each patient. Four weekly assessments were carried out for fundus exploration under 
Table 1. Clinical characteristics of 30 premature infant patients according to the presence of PRH at study's first assessment

\begin{tabular}{|c|c|c|c|c|}
\hline & \multirow{2}{*}{$\begin{array}{c}\text { Total } \\
(\mathrm{n}=30)\end{array}$} & \multicolumn{2}{|c|}{ PRH } & \multirow[t]{2}{*}{$p$} \\
\hline & & Yes $(n=15)$ & No $(n=15)$ & \\
\hline \multicolumn{5}{|l|}{ Gender } \\
\hline Male & $9(30 \%)$ & $4(13 \%)$ & $5(16 \%)$ & 0.028 \\
\hline Female & $21(70 \%)$ & $11(36 \%)$ & $10(33 \%)$ & \\
\hline Age (days) & $33(30-39)$ & $34(30-38)$ & $33(31-39)$ & 0.539 \\
\hline Gestational age (weeks) & $28(27-30)$ & $28(27-30)$ & $29(27-30)$ & 0.967 \\
\hline Birth weight (g) & $1067(645-1725)$ & $945(645-1725)$ & $1100(910-1425)$ & 0.098 \\
\hline Oxygen therapy (days) & $27(14-35)$ & $23(14-35)$ & $28(21-35)$ & 0.081 \\
\hline
\end{tabular}

Values presented as absolute frequencies (percentages) and medians (minimum-maximum).

Table 2. Association of PRH at first study week with the presence of ROP in the following 3 weeks in 30 premature infant patients

\begin{tabular}{|c|c|c|c|c|c|}
\hline & \multirow{2}{*}{$\begin{array}{c}\text { Total } \\
(n=30)\end{array}$} & \multicolumn{2}{|c|}{ PRH at first week } & \multirow[t]{2}{*}{ RR $(95 \% \mathrm{Cl})$} & \multirow[t]{2}{*}{$p$} \\
\hline & & Yes $(n=15)$ & No $(n=15)$ & & \\
\hline \multicolumn{6}{|l|}{ ROP } \\
\hline Week 2 & $23(76 \%)$ & $14(46 \%)$ & $9(30 \%)$ & $1.55(1.00-2.40)$ & 0.040 \\
\hline Week 3 & $25(83 \%)$ & $14(46 \%)$ & $11(36 \%)$ & $1.27(0.91-1.77)$ & 0.165 \\
\hline Week 4 & $17(56 \%)$ & $11(36 \%)$ & $6(20 \%)$ & $1.83(0.91-3.65)$ & 0.070 \\
\hline
\end{tabular}

Values presented as absolute frequencies (percentages), medians (minimum-maximum), relative risk with $95 \% \mathrm{Cl}$ and $P$ value calculated with the Chi-square test.

$\mathrm{PRH}$ : preretinal hemorrhage; ROP: retinopathy of prematurity; $95 \% \mathrm{Cl}$ : $95 \%$ confidence interval.

pharmacologic dilation using tropicamide $(0.8 \%)$ and phenylephrine $(0.5 \%)$, applied every 15 minutes for three times. Two groups were obtained: one first group of patients who had PRH without ROP, and a second group without PRH or ROP at first assessment. Findings obtained at the second, third and fourth weeks were observed and recorded, comparing the presence of $\mathrm{PRH}, \mathrm{ROP}$ and severity thereof, in addition to the indicated treatments in the cases where they were required. For statistical analysis of the results, absolute frequencies and percentages were measured, as well as means or medians; the chi-square test was used for qualitative variables, Student's t-test and Mann-Whitney U-test were used for independent samples, and for the magnitude of association, the relative risk was measured with its $95 \%$ confidence interval. A p-value $<0.05$ was considered to be statistically significant. The SPSS statistical package, version 17.0, was used.

\section{Results}

The study was carried out at the No. 25 UMAE of Monterrey during the months of October and November 2016. Thirty premature patients attending for ROP screening assessment were included, out of which 21 were girls $(70 \%)$ and 9 were boys (30\%); in addition, $376 \%$ of females and $13 \%$ of males had $\mathrm{PRH}$, with a p-value of 0.028 and a statistically significant relationship between the presence of $\mathrm{PRH}$ and patient gender. Median age at assessment was 33 days (range: $30-39$ ), average gestational age was 28 weeks (range: 27-30), average birth weight was $1067 \mathrm{~g}$ (range: 645-1725) and average oxygen therapy time was 27 days (range: 1435) (Table 1).

In the second assessment, a decrease in the incidence of PRH of up to $43.3 \%$ was observed. The development of ROP in 23 patients $(76.7 \%)$ was also evident, with a $p$-value of 0.04 being obtained, with statistical significance. In addition, 19 patients (63.3\%) had seriousness stage I and 4 patients (13.3\%) stage II. At third assessment, the percentage of PRH was not changed and the occurrence of ROP was observed in 25 patients $(83.3 \%)$, with $p=0.165$, without the percentage of seriousness stage I being modified and with the number of patients with stage II increasing to $5(17.7 \%)$, as well as 1 patient with stage III $(3.3 \%)$, which was the highest seriousness level observed during the study. Finally, on the fourth week, PRH was observed in 8 patients $(26.7 \%)$, with a p-value of 0.018 , demonstrating a decrease and resolution in a considerable number of cases in comparison with the study initiation. Seventeen patients $(56.7 \%)$ continued with ROP, with predominance of stage I in 11 of them $(36.7 \%), 9$ patients $(30 \%)$ without ROP with avascular retina and, notoriously, 4 (13.3\%) without ROP with vascularized and fully mature retina (Table 2). 
Relative risk with regard to the presence of $\mathrm{PRH}$ and to the development of ROP was analyzed. In the second week, the relative risk for developing ROP having presented PRH was 1.55 (1.0-2.4), which represented a significant result. Finally, on the fourth week, the relative risk for $\mathrm{PRH}$ was 1.83 (0.91-3.65) (Table 2).

The patients who presented with stage I and II ROP were assessed weekly without receiving medical or surgical treatment. Those at stage III required medical management with intravitreous antiangiogenic agents and close weekly follow-up. Patients with avascular retina without ROP were assessed every 15 days, and those with vascularized retina were discharged. No patients presented with stages IV or V during the study, and treatment with photocoagulation, cryotherapy or surgery was therefore not necessary.

\section{Discussion}

ROP is a condition that is exclusive of premature infants and that manifests itself by a progressive vasoproliferative disorder that generally resolves itself completely; however, there are serious cases that experience important sequels that compromise patient visual function and prognosis, such as vitreous hemorrhage, retinal detachment, neovascular glaucoma and ptisis bulbi, which culminate in an important visual limitation or blindness ${ }^{7,8}$.

ROP occurs with higher prevalence in developing countries, representing an important public health problem. It has a variable global incidence, from $12 \%$ to $78 \%$ of newborns meeting the screening criteria ${ }^{4}$. The importance of screening is reflected in opportune diagnosis and treatment, especially in those infants at initial stages that have not yet developed complications. This screening test should be carried out in every patient with at least 30 days of extrauterine life, with a gestational age shorter than 30 weeks and birth weight lower than $1500 \mathrm{~g}^{7}$. Gestational age of less than than 30 weeks, birth weight lower than $1500 \mathrm{~g}$ and oxygen therapy are the factors mainly associated with the occurrence of this pathology $y^{7,8}$.

The main objective of this study was to compare the presence of $\mathrm{PRH}$ in the assessed patients and the relationship that exists with the development of ROP and its seriousness. Vitreous hemorrhage has been recognized as an initial risk factor for the development and progression of ROP since 1913 by Oguchi' ${ }^{14}$. Different studies have been conducted in order to explain the vitreous hemorrhage pathophysiology in ROP natural evolution, such as the one by Cleary and Ryan ${ }^{15}$, who demonstrated the role of intraocular blood in the development of proliferative retinopathy after penetrating surgery. Ehrenberg et al. ${ }^{16}$ confirmed that pathologic changes occurred even without a previous history of penetrating surgery. Hutcheson et al. ${ }^{15}$ reported in their study an incidence of only $3 \%$ in the assessed infants, but that nevertheless progressed to a borderline high risk stage of disease. So far, no studies comparing the presence of $\mathrm{PRH}$ as a risk factor associated with the development of ROP have been reported, which is why carrying out this work was decided. Among the results obtained in the present study, the incidence of PRH was observed to have values of $43.3 \%$ at first assessments and to be reduced down to $26.7 \%$ by the fourth week; still, it had a statistically significant value, contrasting with the results obtained by Hutchesony for vitreous hemorrhage. The incidence of ROP was $76.6 \%$ at second week, which was regarded as a statistically significant value, with variations throughout the following weeks and showing a final value of $56.7 \%$ in terms of incidence, with statistically non-significant values. Therefore, the presence of $\mathrm{PRH}$ constitutes a risk factor for the onset and development of ROP at initial phases of the disease.

Another objective was to investigate the existing association between the presence of ROP and its seriousness. Stage I was observed to occur more commonly, with a value that ranges from $63.3 \%$ initially to $36.7 \%$ at week 4 , followed by the presence of avascular retina with no data consistent with ROP in $23.3 \%$ to $30 \%$ of total assessed patients at the end of the 4 weeks, with the presentation of stage III standing out as the most serious in $13.3 \%$ of cases. On the other hand, in $13.3 \%$ of patients there was favorable progression until the retina was observed to be free of alterations and with full vascularization, with discharge from the screening program being achieved without further complications. There is considerable difference with the results obtained by Hutcheson et al. in their study, where the majority of patients with vitreous hemorrhage evolved to advanced stage IV and subsequently to retinal detachment.

The criteria for ROP treatment are currently well established and were assessed in the present study ${ }^{8}$. Throughout the work, a weekly evaluation was performed in order for the fundus examination findings to be determined, as well as the indicated treatment in case it was required. At the beginning of the study, $100 \%$ of infants were found to have an immature and 
avascular retina on fundus examination; average age at screening was 33 days of life. At week 2 , there was an important change, with ROP being found in $76.7 \%$, which were classified as stages I and II, without requiring treatment. At third and fourth weeks, progression was evident in four patients who progressed to stage III, and starting medical treatment by applying intravitreous antiangiogenic agents became imminent, with tight follow-up every week was indicated. As previously mentioned, Hutcheson et al. reported that most their patients with vitreous hemorrhage and ROP progressed to stage IV with retinal detachment, and were managed with vitrectomy. Finally, it should be mentioned that, at the end of the study, four patients were discharged from the screening program after demonstrating a favorable response when retinal vascularization was observed, without further need of subsequent follow-up, demonstrating that this pathology resolves in weeks, at an average of 16 weeks after diagnosis.

\section{Conclusions}

The presence of $\mathrm{PRH}$ is significantly associated with the development of ROP and is an independent risk factor for the development of the disease within the first weeks of life; subsequently, it develops even without the presence of $\mathrm{PRH}$, most probably because of other factors.

ROP seriousness manifests itself with mild stages in most cases, without medical treatment or surgical intervention being required, with weekly follow-up, representing a visual prognostic factor that is favorable to the patient.

It is confirmed that, due to its natural evolution, ROP resolves in weeks after diagnosis, and favorably in most cases. In addition, the importance of continuing with the screening at the age established by international ROP guidelines is made evident, in order to decrease the risk of progression, to be able to establish a diagnosis and opportune treatment, and this way avoid complications that affect patient visual prognosis, as well as personal and socioeconomic implications for the patient in the future.

\section{Funding}

The authors declare not having received any funding at all for the performance of the study.

\section{Conflict of interests}

There are no conflicts of interests to declare.

\section{References}

1. Terry TL. Fibroblastic overgrowth of persistent tunica vasculosa lentis in infants born prematurely: II. Report of cases - clinical aspects. Trans Am Ophthalmol Soc. 1942;40:262-84.

2. Shah PK, Prabhu V, Karandikar SS, et al. Retinopathy of prematurity: past, present and future. World J Clin Pediatr. 2016;5:35-46.

3. Ramírez-Ortiz MA, Lara-Molina C, Villanueva-García D, et al. Retinopatía del prematuro: controversias en el uso de antiangiogénicos intraoculares. Bol Med Infant Mex 2013;70:344-50.

4. Flores-Nava G, Barrera-Vázquez C, Fuente-Torres M, et al. Retinopatía del prematuro. Determinación de algunos factores de riesgo. Bol Med Hosp Infant Mex. 2009;66:425-30.

5. Grupo de Trabajo Colaborativo Multicéntrico. Prevención de la ceguera en la infancia por ROP. Retinopatía del prematuro en servicios de neonatología de Argentina. Arch Argent Pediatr. 2006;104:69-74.

6. Gelman R. Diagnosis of plus disease in retinopathy of prematurity using retinal image multiscale analysis. IOVS. 2005;46(12).

7. Fierson WM; American Academy of Pediatrics Section on Ophthalmology; American Academy of Ophthalmology; American Association for Pediatric Ophthalmology and Strabismus; American Association of Certified Orthoptists. Screening examination of premature infants for retinopathy of prematurity. Pediatrics. 2013;131:189-95.

8. Chen J, Stahl A, Hellstrom A, et al. Current update on retinopathy of prematurity: screening and treatment. Curr Opin Pediatr. 2011;23:173-8.

9. McNamara JA, Tasman W, Brown GC, et al. Laser photocoagulation for stage 3+ retinopathy of prematurity. Ophthalmology. 1991;98:576-80.

10. Wu WC, Lien R, Liao PJ, et al. Serum levels of vascular endothelial growth factor and related factors after intravitreous bevacizumab injection for retinopathy of prematurity. JAMA Ophthalmol. 2015; 133:391-7.

11. Shah PK, Narendran V, Kalpana N, et al. Anatomical and visual outcome of stages 4 and 5 retinopathy of prematurity. Eye (Lond). 2009;23:176-80.

12. Mutlu FM, Sarici SU. Treatment of retinopathy of prematurity: a review of conventional and promising new therapeutic options. Int $\mathrm{J}$ Ophthalmol. 2013;6:228-36.

13. Kim MJ, Kim SJ, Yu YS. The risk for retinal detachment associated with hemorrhages pre- and postlaser treatment in retinopathy of prematurity. Retina. 2008;28:1451-7.

14. Oguchi C. Uber die Wirkung von Blutinjektionen in den Glaskorper nebst Bemwerkungen uber die Sogenannte Retinitis proliferans. Von Graefe's Archiv Fur Ophthalmlogie. 1913;84:446-520.

15. Cleary PE, Ryan SJ. Histology of wound, vitreous, and retina in experimental posterior penetrating eye injury in the Rhesus monkey. Am J Ophthalmol. 1979;88:221-31.

16. Hutcheson KA, Nguyen AT, Preslan MW, et al. Vitreous hemorrhage in patients with high-risk retinopathy of prematurity. Am J Ophthalmol. 2003;136:258-63. 